\title{
Energy Expenditure Estimates in Chronic Kidney Disease Using a Novel Physical Activity Questionnaire
}

Sivakumar Sridharan ${ }^{1,2}$

Enric Vilar ${ }^{1,2}$

Sivaramakrishnan Ramanarayanan ${ }^{1}$

Andrew Davenport ${ }^{3}$

Ken Farrington ${ }^{1,2}$

${ }^{1}$ Renal Unit, Lister Hospital, Corey's Mill Lane, Stevenage, UK

${ }^{2}$ University of Hertfordshire, Hatfield, UK

${ }^{3}$ UCL Department of Nephrology, Royal Free Hospital, University College

London, UK

Corresponding Author

Sivakumar Sridharan

Renal Unit

Lister Hospital

Corey's Mill Lane

Stevenage

Hertfordshire

SG1 4AB

UK

Tel: 0044-1438288475

Email: sivakumar.sridharan@nhs.net 


\section{ABSTRACT}

\section{Background:}

Physical activity (PA) levels are low in patients with advanced chronic kidney disease (CKD), and associate with increased morbidity and mortality. Reliable tools to assess PA in CKD are scarce. We aimed to develop and validate a novel PA questionnaire for use in CKD (CKD-PAQ).

\section{Methods:}

In phase 1, a prototype questionnaire was developed based on the validated Recent Physical Activity Questionnaire (RPAQ). Structured feedback on item relevance and clarity was obtained from 40 CKD patients. In phase 2, the questionnaire was refined in 3 iterations in a total of $226 \mathrm{CKD}$ patients against 7-day accelerometer and RPAQ measurements. In phase 3, the definitive CKD-PAQ was compared with RPAQ in 523 CKD patients.

\section{Results:}

In the final iteration of phase 2, CKD-PAQ data were compared to accelerometer-derived and RPAQ data in 60 patients. Mean daily Metabolic Equivalent of Task (MET) and Total Energy Expenditure (TEE) levels were similar by all methods. Intraclass correlation coefficients showed fair agreement (MET) and good (TEE) between accelerometry and both CKD-PAQ and RPAQ. Agreement between questionnaires was excellent. For mean daily MET bias was 0.035 (SD 0.312) for CKD-PAQ and 0.018 (SD 0.326) for RPAQ. For TEE bias was 91 (SD 518) for CKD-PAQ and 44 (SD 548) kcal for RPAQ. Limits of agreement were wide for both parameters, with less dispersion of CKD-PAQ values. In phase 3, agreement between questionnaires was good (MET) and excellent (TEE). Bias of CKD-PAQ-derived mean daily MET from RPAQ-derived values was 0.031 (SD 0.193) with $95 \%$ limits of agreement -0.346 to 0.409 . Corresponding values for TEE were 48 (SD $325)$ and -588 to $685 \mathrm{kcal} /$ day. CKD-PAQ appeared to improve discrimination between low activity groups.

\section{Conclusions:}

CKD-PAQ performs comparably to RPAQ though is shorter, easier to complete, may better capture low level activity and improve discrimination between low-activity groups.

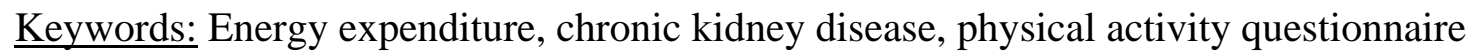

Word count

Abstract 300

Body of Text 3055 
What is already known about the subject?

- Physical activity levels are substantially low in CKD patients. Reduced physical functioning capacity and increased fatigue levels contribute to low physical activity levels in these patients. Estimating physical activity is vital to nutritional management of CKD patients.

- Except for one recently developed questionnaire (LoPAQ), none of the existing physical activity questionnaires are developed specifically in individuals with CKD. However, LoPAQ was developed only in dialysis patients and did not include non-dialysis CKD and transplant patients.

- A disease-specific physical activity measurement tool will increase accuracy and applicability of such tools for nutritional and dialysis management.

What this study adds?

- This is the first study to develop a CKD-specific physical activity measurement tool across the spectrum of patients with CKD

- The CKD-PAQ questionnaire performs comparably, if not slightly better, to the existing validated questionnaires but has some advantages over the existing ones.

- This novel questionnaire is shorter compared to existing questionnaires, easier for patients to complete, focuses on activities commonly performed by this patient population and may better capture low level activity commonly prevalent in CKD patients

What impact this may have on practice?

- When validated in other larger cohorts, this novel CKD-PAQ questionnaire can be easily used in every day clinical practice for nutritional management of CKD patients.

- Total energy expenditure estimated from the novel questionnaire can also be used in conjunction with other clinical data for appropriate dialysis management. 


\section{INTRODUCTION}

Physical activity (PA) levels have been shown to be low in individuals with chronic kidney disease $(\mathrm{CKD})$ and in those receiving kidney replacement therapy [1-4]. There are clear benefits, including improved survival and quality of life, in patients with higher PA levels

[5-7]. Successful implementation of any programme aiming to encourage PA in this patient population depends on reliable tools to assess PA consistently.

The use of doubly labelled water or accelerometers for PA measurement is not feasible in routine clinical practice. Self-report activity questionnaires are a practical alternative. None of the PA questionnaires used in studies involving patients with CKD have been derived from this patient population. Most of these questionnaires are derived from young healthy adults and as such, may not be applicable to specific patient groups. CKD is predominantly a disease of the elderly and these CKD questionnaires may not be valid in this patient population. A study examining the validity of ten PA questionnaires in elderly individuals in general population against doubly labelled water found that few questionnaires were reliable for use in elderly [8]. Moreover, the individual variability was high for all the questionnaires which limits their use in these individuals.

Most of the existing questionnaires focus on moderate to vigorous PA and were not designed for studying PA levels in populations with low level physical activity. There is evidence to suggest PA levels in dialysis patients are lower than healthy age-matched controls with no regular physical activity [4]. There is a dearth of reliable tools to measure PA level in populations such as CKD patients who are predominantly elderly with low level PA. 
Recent Physical Activity Questionnaire (RPAQ) has been validated in individuals with CKD using doubly labelled water measurements [9]. However, it showed that the questionnaire was not reliable in capturing low intensity and sedentary activities. This reinforces the need for developing a novel physical activity questionnaire for better measurement of PA in CKD.

Our aim in this study was to develop and validate a novel physical activity questionnaire specifically designed for individuals with CKD (CKD-PAQ) using accelerometer derived physical activity measurements.

\section{SUBJECTS AND METHODS}

\section{Ethical Review}

The study was approved by the National Research Ethics service. All subjects gave written informed consent to take part.

\section{Subjects}

Patients older than 18 years with CKD stages 1-5 including those receiving dialysis and those with functioning kidney transplant were recruited. Those who, in the judgement of the clinical team, had insufficient capacity or insufficient understanding of English to allow valid consent, were not approached for inclusion in the study by the study team.

\section{Study Protocol}

The study was carried out in three phases (i) an initial qualitative phase consisting of structured patients interviews-(ii) a development phase in which the questionnaire was modified sequentially to improve reliability and accuracy of energy expenditure estimation 
in comparison with accelerometer estimates and (iii) a final phase to compare energy estimates from the novel questionnaire against existing validated PA questionnaire. A flowchart depicting the study design is shown in Figure 1. There was no overlap of study participants across the different phases of the study.

\section{Development of the questionnaire}

A novel physical activity questionnaire (CKD-PAQ) was developed based on the Recent Physical Activity Questionnaire (RPAQ). In the initial phase, 40 patients with CKD including dialysis and transplant patients, were recruited to complete the first prototype questionnaire. Structured feedback was obtained through one-to-one interviews with each of the participant focusing on the clarity of questionnaire items, ease of completion and on the breadth of activities captured by the questionnaire. This feedback was then used to develop the first iteration of the questionnaire to be tested against accelerometer measured PA.

The second phase of the development of the questionnaire was conducted through 3 stages. The questionnaire was iterated at the end of each of the first two stages to improve capture of different levels of activity compared to measured PA from an accelerometer. The questionnaire items were modified to achieve this objective in the first two stages. The initial two versions of the questionnaire included an exhaustive list of leisure and work activities. However, on review of participant responses and the contribution of some of the activities to the final model for energy expenditure estimation compared to the accelerometer measures, some of the activities were removed from subsequent iterations. Some of the questionnaire items were also modified to improve clarity and for ease of analysis. The final version of the questionnaire (Supplementary materials) at the end of the 
third stage was then employed in the final phase in a different cohort of patients for comparison of energy estimates against the validated RPAQ questionnaire.

CKD-PAQ and RPAQ both enquire regarding activities performed at home, at work and recreational activities over the preceding 4 weeks. However, CKD-PAQ is much shorter (27 items) compared to RPAQ (55 items). For haemodialysis (HD) patients, CKD-PAQ has an additional 5 items to collect information regarding their dialysis sessions. CKDPAQ focuses on simple range of recreational activities compared to RPAQ which contains a comprehensive list of high intensity activities which are not commonly carried out by individuals with CKD.

\section{Data collection}

The following data were collected on all participants.

1. Demographic and anthropometric data including height and weight, and residence postcode. The English Index of Multiple Deprivation was calculated using the participants' postcode.

2. Comorbidity data, which was used to calculate Charlson Comorbidity Index

3. PA assessment using questionnaires and accelerometer

\section{Measurement of physical activity}

Physical activity was measured using a wrist-worn accelerometer (GT9X Link, ActiGraph LLC, Florida, USA). Participants were advised to wear the accelerometer on the nondominant wrist for 24 hours a day for 7 consecutive days. At the end of the measurement period, the accelerometer data was retrieved through the ActiLife software for analysis. The data included total vector magnitude counts, steps per minute and mean daily 
Metabolic Equivalent of Task (MET) amongst other raw movement related measures. The rate of energy spent during any PA is expressed as MET value. One MET is the energy spent sitting at rest and is approximately equal to $1 \mathrm{kcal} / \mathrm{hr} / \mathrm{kg}$ of body weight. The daily MET from the measured activity was used to calculate Total Energy Expenditure (TEE) as described below.

\section{Physical activity assessment}

At the end of the 7-day study period, subjects completed two PA questionnaires - RPAQ and CKD-PAQ. RPAQ is a validated questionnaire which enquires about various activities performed at home, work and at leisure time and the time spent in each of those activities over the preceding 4 weeks [10]. RPAQ has been validated in CKD patients for energy expenditure estimation using doubly labelled water [9].

\section{Estimation of TEE}

Accelerometery

TEE was calculated using the following relationship $T E E(k c a l / d a y)=R E E(k c a l / d a y) \times$ Mean Daily MET $\quad$ Equation 1 where REE is Resting energy expenditure estimated from a previously published diseasespecific equation [11] and Mean Daily MET is an output variable from the measured PA by accelerometery.

\section{Physical Activity Questionnaires}

Energy expenditure estimation from RPAQ was carried out as previously described [9]. Briefly, a MET (Metabolic Equivalent of Task) value was assigned to each reported 
activity as per the Compendium of Physical Activities which was then used to calculate the Mean Daily MET as shown below.

$$
\text { Mean Daily MET }=\frac{\text { Total Daily } M E T}{24}
$$

\section{Equation 2}

The unaccounted time from the questionnaire was assigned a MET of 1.3 as previously published [9]. TEE was then calculated using the relationship depicted in Equation 1. Energy expenditure estimation from CKD-PAQ was carried out in the same manner as that used for RPAQ.

\section{Statistical Analysis}

Statistical analysis was carried out using SPSS ${ }^{\circledR}$ version 26 (SPSS Software, IBM Corporation, Armonk, NY, USA) and PRISM 9 (Graphpad Software LLC). Based on previous data on correlation of energy estimation from RPAQ and measured TEE, a sample size of 40 in each phase was considered to be sufficient to establish significant intragroup correlations, assuming $\alpha=0.05$ and for power of 0.8 . A sample size of 400 for the final phase was considered to provide sufficient power. Normally distributed data are presented as mean \pm standard deviation and non-normally distributed data as median (interquartile range). The significance of differences between means was determined using Student's t-test and of medians by Mann-Whitney U-test. For phase 2 data, comparison was made between MET and TEE values derived from accelerometry (METACC and

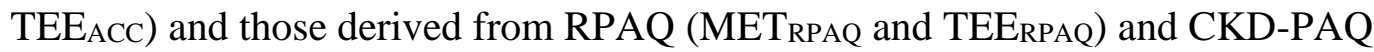
(MET ${ }_{\mathrm{CKD}}$ and $\mathrm{TEE}_{\mathrm{CKD}}$ ) by calculating the relevant intraclass correlation coefficient (ICC) and by Bland-Altman analysis. For phase 3 data similar comparisons were made between the questionnaire derived parameters. . A p-value of $<0.05$ was considered significant. 


\section{$\underline{\text { RESULTS }}$}

266 patients were recruited in the development phases, 40 in the initial qualitative phase, and 226 in the remaining 3 stages of the development. The number of participants in each stage was 89,77 and 60 respectively. The results presented are from the final iteration involving 60 patients. This version was used for the final phase involving 523 patients, 394 of whom completed both questionnaires. Demographic and biochemical characteristics for both cohorts are shown in Table 1.

\section{Development phase}

Median values for METACC, METRPAQ, and METCKD were similar [1.35 (0.26), 1.26 ((0.27), and $1.31(0.33)$ respectively]. There were no significant differences between $\operatorname{MET}_{A C C}$ and either METRPAQ $(p=0.08)$ or $\operatorname{MET}_{C K D}(p=0.084)$ nor between METRPAQ and $\operatorname{MET}_{C K D}(p=0.287)$. Likewise mean values for TEEACC, TEERPAQ and TEECKD were similar $(2379 \pm 630,2413 \pm 873,2361 \pm 827$ kcal respectively $)$, and there were no differences between TEEACC and either TEERPAQ $(p=0.561)$ or TEECKD $(p=0.203)$ nor between TEERPAQ and TEECKD $(\mathrm{p}=0.598)$.

There was fair agreement between $\mathrm{MET}_{\mathrm{ACC}}$ and both $\mathrm{MET}_{\mathrm{RPAQ}}[\mathrm{ICC}=0.441(0.031-0.677$ : $\mathrm{p}=0.019)$ and $\mathrm{MET}_{\mathrm{CKD}}[\mathrm{ICC}=0.455(0.059-0.685): \mathrm{p}=0.015]$ and excellent agreement between MET $_{\text {RPAQ }}$ and MET $_{\text {CKD }}[\mathrm{ICC}=0.905(0.836-0.944): \mathrm{p}<0.001]$. Agreement was good between TEEACC and both TEERPAQ $[$ ICC $=0.789(0.636-0.878: p<0.001)$ and TEE $_{C K D}[\mathrm{ICC}=0.751(0.572-0.855: \mathrm{p}<0.001]$ and excellent between TEERPAQ and TEECKD $[\mathrm{ICC}=0.917(0.857-0.951): \mathrm{p}<0.001]$ 
Table 2 shows the results of Bland-Altman analysis for comparisons of mean daily MET and TEE from questionnaires and from accelerometry. Bias for both parameters was small and slightly lower for RPAQ derived parameters. However, both the standard deviation of the bias and the $95 \%$ limits of agreement showed less dispersion for CKD-PAQ than for RPAQ. Figure 2 shows the Bland-Altman plot of TEE derived from accelerometry (TEEACC) and that from CKD-PAQ (TEECKD). Bland-Altman comparisons of METCKD and METRPAQ, and TEE $_{C K D}$ and TEERPAQ showed minimal bias and even less dispersion (Table 2).

The relationship between $\mathrm{TEE}_{\mathrm{ACC}}$ and tertiles of TEECKD and TEERPAQ respectively are shown in Figures $3 \mathrm{~A}$ and 3B. There was a significant difference in TEEACC levels between both middle and upper TEECKD tertiles compared to the lowest tertile. For TEERPAQ, the only significant difference in TEEACC was between the lowest and highest TEERPAQ tertile.

In a multivariable regression model of TEE $_{A C C}$ (Table 3), METCKD was a significant predictor after adjustment for age, sex. body surface area and comorbidity (adjusted R square 0.719). Substituting METRPAQ for MET $_{\mathrm{CKD}}$ in the model gave similar results (adjusted $\mathrm{R}$ square 0.703$)$.

\section{Final phase}

The final version of CKD-PAQ questionnaire was compared against RPAQ in a larger cohort of 523 CKD patients (Table 1). Median unaccounted time was lower with CKD-PAQ than RPAQ (1.0 vs 9.8 hours, $\mathrm{p}<0.001)$. Mean MET $_{\text {CKD }}$ was slightly lower than mean MET RPAQ $(1.24 \pm 0.28$ vs $1.27 \pm 0.23: \mathrm{p}=0.001)$. ICC for the comparison showed good agreement

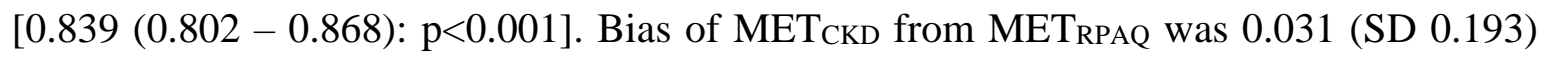
with $95 \%$ limits of agreement -0.346 to 0.409 . Mean TEE $E_{C D D}$ was lower than TEERPAQ (1964 
\pm 643 vs $2012 \pm 580 \mathrm{kcal} /$ day, $\mathrm{p}<0.001)$. ICC for the comparison showed excellent agreement [0.923 (0.905-0.937): $\mathrm{p}<0.001]$. Bias was 48 (SD 325) with limits of agreement -588 to $685 \mathrm{kcal} / \mathrm{day}$.

Women had a lower MET RPAQ $(1.23$ vs $1.29, \mathrm{p}=0.001)$ but not MET $\mathrm{CKD}$, than men. Younger patients (< 65 years) had higher TEECKD (2183 vs $1689 \mathrm{kcal} /$ day, p < 0.001) and TEERPAQ (2238 vs $1738 \mathrm{kcal} / \mathrm{day}, \mathrm{p}<0.001)$ than older counterparts. Charlson Comorbidity Index correlated negatively with TEE TKD $(\mathrm{r}=-0.269, \mathrm{p}<0.001)$ but not TEERPAQ. Both MET $\mathrm{CKD}$ $($ rho $=0.232, \mathrm{p}<0.001)$ and MET $_{\text {RPAQ }}$ RPAQ (rho $\left.=0.180, \mathrm{p}<0.001\right)$ correlated with deprivation index and both TEE $E_{C K D}(1864$ vs. $2046 \mathrm{kcal} /$ day, $\mathrm{p}=0.002)$ and TEERPAQ $(1921$ vs. $2091 \mathrm{kcal} / \mathrm{day}, \mathrm{p}=0.002$ ) were lower in participants living in most deprived areas (deprivation index $<$ median).

There were significant differences in both mean daily MET and TEE levels between modality groups (Table 4). Compared with in-centre HD patients both these parameters from both questionnaires were higher in CKD and Transplant patients. CKD-PAQ, but not RPAQ, derived levels of both parameters were higher in home than in in-centre HD patients significant for TEE. Transplant patients were younger and had lower Charlson scores than in-centre HD patients. CKD patients had lower Charlson scores and were less deprived than in-centre HD patients. Home HD patients were also less deprived (Supplementary table).

\section{DISCUSSION}

The primary aim of this study was to develop and validate a novel PA questionnaire specifically in individuals with CKD. The study showed that the energy estimates from the novel questionnaire, CKD-PAQ provided acceptable estimations of accelerometer-based 
parameters and performed similarly to the existing validated RPAQ questionnaire. The novel CKD-PAQ questionnaire also has the advantage of being substantially shorter and simpler to complete than RPAQ.

Routine use of accelerometers is not practical in day-to-day clinical practice. Physical activity questionnaires are useful alternatives for estimation of PA and energy expenditure. Developing a PA questionnaire in CKD population poses some challenges. Patients with CKD are more likely to be elderly and have higher comorbidity and hence, questionnaires developed in younger people may not be reliable when used in CKD population $[12,13]$. As CKD patients are more likely to have low levels of PA, it is vital that any diseasespecific PA questionnaire is able to capture low intensity activity to enable accurate assessment. Besides a recently developed LoPAQ questionnaire in dialysis patients [14, 15], none of the existing PA questionnaires have been developed in CKD patient population.

Analysis of standard correlations is not an appropriate method to assess the agreement between methods [16-18]. In the first instance we calculated ICC levels. These showed fair agreement between mean daily MET from both CKD-PAQ and from RPAQ with accelerometer measures. For TEE agreement in both cases was good. Agreement between CKD-PAQ and RPAQ derived parameters was excellent in phase 2 and performed similarly in the phase 3 cohort. CKD-PAQ derived MET adjusted for age, sex, body surface area was an independent predictor of measured TEE - comparable to RPAQ derived MET (Table 3). We also used the Bland-Altman technique to compare mean daily MET and TEE estimated from CKD-PAQ and RPAQ to that measured by accelerometry. Mean bias between the accelerometer and both questionnaires was small though slightly 
lower for RPAQ. However, the limits of agreement were quite wide - though slightly less for CKD-PAQ - and perhaps related to differences in the assessment time periods - 7-days in the accelerometer study and 4 weeks for both questionnaires [19]. All these finding suggest comparable performance of the CKD-PAQ and RPAQ questionnaires.

In addition, we found better discrimination between lower and middle accelerometer derived TEE tertiles with CKD-PAQ derived TEE than with RPAQ derived TEE. We also found significant differences in energy expenditure levels between in-centre HD and home HD patients with CKD-PAQ derived values but not with RPAQ values. Both these findings suggest that CKD-PAQ derived energy expenditure values capture low intensity activities better than RPAQ derived values. There may be a number of reasons for this, including that CKD-PAQ is simpler to complete than RPAQ and thus may be more likely to be completed accurately. However, another major difference is the length of unaccounted time, i.e. the number of hours in a day that are not captured by the questionnaires, which is significantly lower with CKD-PAQ. In the final phase of the study, the median unaccounted time with CKD-PAQ was 1 hour per day compared to more than 9 hours with RPAQ. This demonstrates much more complete activity data capture with the novel questionnaire. As data capture is more complete, there is less risk of overestimating PA level with CKD-PAQ as evidenced by significantly lower MET and TEE with CKD-PAQ compared to RPAQ. Hence there may be better discrimination between assessed PA levels in groups, such as those across the range of CKD, with low activity levels.

This study has its limitations. As with any questionnaire-based study, recall bias may have been a confounding factor. Although this has been minimised to some extent by enquiring 
about specific activities, some activities especially the low intensity ones, may not have been accurately reported by participants. The use of accelerometry in CKD patient population with low levels of PA may also limit the accuracy of the accelerometer data as these are designed predominantly to measure PA levels and not sedentary lifestyle. However, the gold standard doubly labelled water method of measuring total energy expenditure is not always feasible due to high costs and clinical problems with water turnover in CKD (especially dialysis) patients, leaving accelerometery as the most practical alternative "gold standard".

There are a number of potential benefits in deploying CKD-PAQ in routine clinical practice. Physical activity measurement in patients with CKD could be useful in their nutritional management by providing energy expenditure estimation and help identify patients with declining physical functioning. As CKD-PAQ focuses on common and routine physical activities, it can provide insight into potential target areas to increase PA levels and physical functioning, and can also be used to assess response to PA related interventions.

In conclusion, this is the first study to have developed a PA questionnaire in individuals across the range of CKD. This study has shown that CKD-PAQ is a valid tool for assessment of PA in CKD patients and performs comparably to the existing validated RPAQ questionnaire. CKD-PAQ may capture low level PA more completely and better discriminate between groups with habitually low PA - such as the CKD population. The novel CKD-PAQ questionnaire needs further validation in larger cohort of patients with CKD. 


\section{Funding Support}

This research was partly funded by a joint research grant from British Renal Society and Kidney Care UK.

\section{$\underline{\text { Author's contributions }}$}

SS - Designing and setting up the study, collection and interpretation of data, drafting and revising the manuscript, approving the final version EV - Collection and interpretation of the data, revised and approved the final version of the manuscript SR - Collection and interpretation of the data, revised and approved the final version of the manuscript $\mathrm{AD}$ - Collection and interpretation of the data, revised and approved the final version of the manuscript, provided intellectual content of critical importance to the article $\mathrm{KF}$ - Concept of study, interpretation of the data, revised and approved the final version of the manuscript, provided intellectual content of critical importance to the article

\section{Conflict of interest statement: None declared}

Data availability: The data underlying this article will be shared on reasonable request to the corresponding author. 


\section{REFERENCES}

1. Baria F, Kamimura MA, Avesani CM, et al. Activity-related Energy Expenditure of Patients Undergoing Hemodialysis. Journal of Renal Nutrition 2011;21(3):226-234

2. Hawkins MS, Sevick MA, Richardson CR, et al. Association between Physical Activity and Kidney Function: National Health and Nutrition Examination Survey. Medicine and Science in Sports and Exercise 2011;43(8):1457-1464

3. Robinson-Cohen C, Littman AJ, Duncan GE, et al. Assessment of physical activity in chronic kidney disease. J Ren Nutr 2013;23(2):123-131

4. Johansen KL, Chertow GM, Ng AV, et al. Physical activity levels in patients on hemodialysis and healthy sedentary controls. Kidney International 2000;57(6):2564-2570

5. O'Hare AM, Tawney K, Bacchetti P, Johansen KL. Decreased survival among sedentary patients undergoing dialysis: results from the dialysis morbidity and mortality study wave 2. Am J Kidney Dis 2003;41(2):447-454

6. Stack AG, Molony DA, Rives T, Tyson J, Murthy BV. Association of physical activity with mortality in the US dialysis population. Am J Kidney Dis 2005;45(4):690-701

7. Suh MR, Jung HH, Kim SB, Park JS, Yang WS. Effects of regular exercise on anxiety, depression, and quality of life in maintenance hemodialysis patients. Ren Fail 2002;24(3):337-345

8. Bonnefoy M, Normand S, Pachiaudi C, et al. Simultaneous Validation of Ten Physical Activity Questionnaires in Older Men: A Doubly Labeled Water Study. Journal of the American Geriatrics Society 2001;49(1):28-35

9. Sridharan S, Wong J, Vilar E, Farrington K. Comparison of energy estimates in chronic kidney disease using doubly-labelled water. J Hum Nutr Diet 2016;29(1):59-66

10. Besson H, Brage S, Jakes RW, Ekelund U, Wareham NJ. Estimating physical activity energy expenditure, sedentary time, and physical activity intensity by self-report in adults. American Journal of Clinical Nutrition 2010;91(1):106-114

11. Vilar E, Machado A, Garrett A, et al. Disease-specific predictive formulas for energy expenditure in the dialysis population. J Ren Nutr 2014;24(4):243-251

12. Washburn RA, Jette AM, Janney CA. Using Age-Neutral Physical Activity Questionnaires in Research with the Elderly. Journal of Aging and Health 1990;2(3):341356

13. Washburn RA, Smith KW, Jette AM, Janney CA. The Physical Activity Scale for the Elderly (PASE): development and evaluation. J Clin Epidemiol 1993;46(2):153-162

14. Johansen KL, Painter P, Delgado C, Doyle J. Characterization of physical activity and sitting time among patients on hemodialysis using a new physical activity instrument. J Ren Nutr 2015;25(1):25-30

15. Kittiskulnam P, Sheshadri A, Johansen KL. Validation of a New Physical Activity Instrument Against Pedometers Among Dialysis Patients. J Ren Nutr 2019;29(6):498-503

16. Bellach B. Remarks on the use of Pearson's correlation coefficient and other association measures in assessing validity and reliability of dietary assessment methods. Eur J Clin Nutr 1993;47 Suppl 2:S42-45

17. Bland JM, Altman DG. Statistical methods for assessing agreement between two methods of clinical measurement. Lancet 1986;1(8476):307-310

18. Schmidt ME, Steindorf K. Statistical methods for the validation of questionnairesdiscrepancy between theory and practice. Methods Inf Med 2006;45(4):409-413

19. Neilson HK, Robson PJ, Friedenreich CM, Csizmadi I. Estimating activity energy expenditure: how valid are physical activity questionnaires? American Journal of Clinical Nutrition 2008;87(2):279-291 
Table 1: Demographic characteristics of Study Participants.

BSA: Body surface area, BMI: Body Mass Index; CCI: Charlson Comorbidity Index; REE: Resting Energy Expenditure; CKD: Chronic kidney disease Stage 3-5; HD: haemodialysis; PD: Peritoneal Dialysis

\begin{tabular}{|l|c|c|}
\hline & Development phase $(\mathrm{n}=60)$ & Final phase $(\mathrm{n}=523)$ \\
\hline Age $($ years $)$ & $58.3 \pm 15.1$ & $60.8 \pm 16.1$ \\
\hline Males $(\%)$ & 57.6 & 63.7 \\
\hline Weight $(\mathrm{kg})$ & $86.6 \pm 22.1$ & $76.5 \pm 18.9$ \\
\hline Height $(\mathrm{cm})$ & $169.6 \pm 9.5$ & $168.2 \pm 10.6$ \\
\hline BSA $\left(\mathrm{m}^{2}\right)$ & $2.03 \pm 0.3$ & $1.89 \pm 0.27$ \\
\hline BMI $\left(\mathrm{kg} / \mathrm{m}^{2}\right)$ & $30.0 \pm 6.7$ & $27.0 \pm 6.2$ \\
\hline CCI & $4.2 \pm 2.5$ & $4.9 \pm 2.4$ \\
\hline REE $(\mathrm{kcal} / \mathrm{day})$ & $1706 \pm 283$ & $1577 \pm 258$ \\
\hline CKD & 20 & 24 \\
\hline In-Centre HD & 24 & 436 \\
\hline Home HD & 1 & 20 \\
\hline PD & 0 & 9 \\
\hline Transplant & 15 & 34 \\
\hline
\end{tabular}


Table 2. Bland-Altman comparisons between physical activity measures derived from accelerometry and CKD-PAQ and RPAQ questionnaires.

$\mathrm{SD}=$ standard deviation. $\mathrm{LOA}=$ Limits of Agreement

\begin{tabular}{|l|c|c|}
\hline & CKD-PAQ derived & RPAQ derived \\
\hline Bland-Altman comparisons with Accelerometer & \\
\hline Mean daily MET & & 0.018 \\
\hline Bias & 0.035 & 0.326 \\
\hline SD Bias & 0.312 & -0.656 to 0.621 \\
\hline 95\% LOA & -0.646 to 0.577 & 44 \\
\hline TEE & & 548 \\
\hline Bias & 91 & -1030 to 1117 \\
\hline SD Bias & 518 & \\
\hline 95\% LOA & -925 to 1108 & \\
\hline Bland-Altman comparisons with RPAQ & \\
\hline Mean daily MET & & \\
\hline Bias & 0.008 & \\
\hline SD Bias & 0.265 & \\
\hline 95\% LOA & -0.512 to 0.527 & \\
\hline TEE & & \\
\hline Bias & 34 & \\
\hline SD Bias & 481 & \\
\hline 95\% LOA & -909 to 978 & \\
\hline
\end{tabular}


Table 3: Multivariate linear regression model of independent predictors of accelerometer measured TEE. Adjusted $\mathrm{R}^{2}=0.719$; MET $_{\mathrm{paq}}$ : Mean daily MET from CKD-PAQ, BSA: Body surface area

\begin{tabular}{|l|c|c|c|c|c|}
\hline & \multicolumn{2}{|c|}{$\begin{array}{c}\text { Unstandardised } \\
\text { Coefficients }\end{array}$} & $\begin{array}{c}\text { Standardised } \\
\text { Coefficients }\end{array}$ & \multirow{2}{*}{ t } & p-value \\
\cline { 2 - 5 } & B & $\begin{array}{c}\text { Std. } \\
\text { Error }\end{array}$ & Beta & & \\
\hline (Constant) & -1048.66 & 387.63 & & -2.705 & 0.009 \\
\hline Age $\geq \mathbf{6 5}$ years & -453.37 & 96.79 & -0.354 & -4.684 & $<0.001$ \\
\hline Sex & -207.34 & 98.77 & -0.164 & -2.099 & 0.041 \\
\left.\hline BSA ${\left(\mathbf{m}^{2}\right)}^{\mathbf{2}}\right)$ & 1708.45 & 181.05 & 0.761 & 9.436 & $<0.001$ \\
\hline Log MET $_{\text {paq }}$ & 909.73 & 258.59 & 0.266 & 3.518 & 0.001 \\
\hline
\end{tabular}


Table 4: Mean daily MET and TEE derived from CKD-PAQ and RPAQ questionnaires in different modalities.

ICHD: In centre haemodialysis, PD: peritoneal dialysis, HHD: home haemodialysis, Transplant: patients with functioning kidney transplant, CKD: patients with stage 3-5 chronic kidney disease. $\mathrm{P}$ values indicate significance of differences of mean values of parameters in other modalities from mean levels in patients receiving in-centre haemodialysis using one-way ANOVA with Bonferroni method of post-hoc testing.

\begin{tabular}{|c|c|c|c|c|c|c|c|}
\hline & \multicolumn{3}{|c|}{ Mean daily MET } & \multicolumn{3}{|c|}{ TEE } \\
\hline & & $\mathbf{N}$ & Mean \pm SD & p-value & $\mathbf{N}$ & Mean \pm SD & p-value \\
\hline \multirow{5}{*}{$\begin{array}{l}\text { CKD- } \\
\text { PAQ }\end{array}$} & ICHD & 384 & $1.18 \pm 0.23$ & Reference & 383 & $1860 \pm 514$ & Reference \\
\hline & PD & 9 & $1.18 \pm 0.15$ & 1.00 & 9 & $1774 \pm 357$ & 1.00 \\
\hline & HHD & 19 & $1.35 \pm 0.27$ & 0.079 & 19 & $2299 \pm 648$ & 0.014 \\
\hline & Transplant & 33 & $1.48 \pm 0.28$ & $<0.001$ & 32 & $2535 \pm 674$ & $<0.001$ \\
\hline & CKD & 24 & $1.46 \pm 0.56$ & $<0.001$ & 16 & $2691 \pm 1422$ & $<0.001$ \\
\hline \multirow{5}{*}{ RPAQ } & ICHD & 360 & $1.24 \pm 0.20$ & Reference & 359 & $1940 \pm 491$ & Reference \\
\hline & PD & 9 & $1.21 \pm 0.10$ & 1.00 & 9 & $1823 \pm 335$ & 1.00 \\
\hline & HHD & 18 & $1.30 \pm 0.20$ & 1.00 & 18 & $2152 \pm 540$ & 1.00 \\
\hline & Transplant & 30 & $1.44 \pm 0.21$ & $<0.001$ & 29 & $2494 \pm 565$ & $<0.001$ \\
\hline & CKD & 22 & $1.46 \pm 0.57$ & $<0.001$ & 14 & $2657 \pm 1490$ & $<0.001$ \\
\hline
\end{tabular}


Figure 1: Flowchart depicting the study design

Figure 2: Bland-Altman plot showing bias and limits of agreement between TEE $_{\mathrm{ACC}}$ and TEE $_{\mathbf{C K D}}$. Difference between TEE measured by accelerometer and CKD-PAQ plotted against the mean of the two measurements. A negative sign indicates an overestimation and a positive sign indicates an underestimation by the questionnaire.

Figure 3: A plot of accelerometer measured TEE against (A) CKD-PAQ TEE tertiles (B) RPAQ TEE tertiles 